\title{
Disposition Start Time
}

National Cancer Institute

\section{Source}

National Cancer Institute. Disposition Start Time. NCI Thesaurus. Code C83131.

The time at which the disposition event begins. 